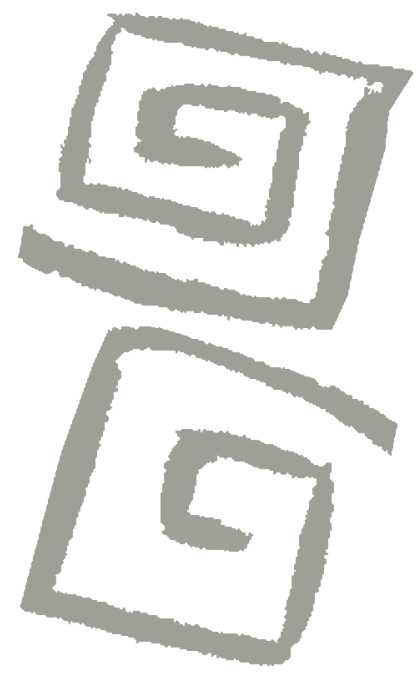

\title{
Explotación sexual comercial de niños, niñas y adolescentes: significados y prácticas de trabajadores/as y residentes del centro de Medellín (Colombia), 2015
}

\author{
Commercial sexual exploitation of children: Meanings \\ and practices of people who work and reside in \\ downtown Medellin (Colombia), 2015
}

Diego Alejandro Ossa-Estrada ${ }^{1}$, Iván Felipe Muñoz-Echeverri ${ }^{2}$

${ }^{1}$ Profesional en Gerencia de Sistemas de Información en Salud. Estudiante, Maestría en Salud Pública, Universidad Nacional de Colombia, Bogotá, Colombia. $\bowtie$ iD

${ }^{2}$ Candidato a Doctor en Salud Pública. DocenteInvestigador, Facultad Nacional de Salud Pública, Universidad de Antioquia, Medellín, Colombia. $\bowtie$ (iD
RESUMEN La explotación sexual comercial es un problema de salud pública y una vulneración de los derechos de niños, niñas y adolescentes. La respuesta a este problema se ha visto afectada por los significados y las prácticas de los actores implicados. Como aporte a una mejor comprensión del problema, entre 2014 y 2015 se realizó una investigación social cualitativa, con el enfoque de la teoría fundamentada, cuyo objetivo fue comprender los significados y las prácticas sobre esta problemática de las personas que permanecen en un sector del centro de la ciudad con alta presencia de explotación sexual comercial de niños, niñas y adolescentes. Las técnicas usadas fueron observación participante y entrevista semiestructurada. Encontramos que predominan concepciones que conducen a prácticas que agravan y perpetúan la vulneración de derechos. Aunque también se identificaron prácticas de protección a las víctimas, estas son limitadas por asuntos críticos del contexto. Las acciones para erradicar la explotación sexual deben trabajar con la comunidad sus significados para potenciar las prácticas protectoras que realizan y reducir las barreras.

PALABRAS CLAVES Abuso Sexual Infantil; Violaciones de los Derechos Humanos; Maltrato a los Niños; Defensa del Niño; Investigación Cualitativa; Colombia.

\begin{abstract}
The commercial sexual exploitation of children is a public health problem and a serious violation of the rights of children and adolescents. The response to this problem has been affected by the meanings and practices of the actors involved. In order to contribute to a better understanding of the problem, a qualitative social study using a grounded theory approach was carried out between 2014 and 2015. The aim was to understand the meanings and practices regarding this issue of people who spend time in an area of the city center with a high presence of commercial sexual exploitation of children and adolescents. The techniques used were participant observation and semistructured interviews. We found that the predominate conceptions lead to practices that aggravate and perpetuate rights violations. Although practices of protection towards victims were identified, these were limited to critical aspects of the context. Actions to eradicate commercial sexual exploitation should work with the community and the meanings within the community regarding sexual exploitation so as to potentiate the victim protection practices carried out and reduce barriers to such practices.
\end{abstract}

KEY WORDS Child Abuse, Sexual; Human Rights Abuses; Child Abuse; Child Advocacy; Qualitative Research; Colombia. 


\section{INTRODUCCIÓN}

La explotación sexual comercial de niños, niñas y adolescentes incluye todas las actividades que una persona pueda hacer con el fin de usar el cuerpo de un niño, niña o adolescente para sacar provecho de carácter sexual ${ }^{(1)}$. $\mathrm{Ha}$ sido reconocida a nivel internacional como una de las peores formas de trabajo infantil(2); una forma de violencia sexual ${ }^{(3)}$; una grave vulneración de derechos de niños, niñas y adolescentes $^{(4,5)}$; una conducta delictiva ${ }^{(6)}$, en la que son tratados como un objeto sexual y una mercancía por los diferentes tipos de explotadores ${ }^{(7,8,9,10,11)}$. En materia de salud pública, la gravedad de la problemática se fundamenta en las graves consecuencias para la salud física, situación nutricional, salud sexual y reproductiva, salud mental y bienestar social de las víctimas ${ }^{(12,13,14,15,16,17,18,19,20,21,22,23,24)}$.

Al respecto, se han reconocido varias manifestaciones o modalidades: utilización en pornografía, explotación sexual comercial en el marco de viajes y turismo, trata con fines sexuales, matrimonios forzados, reclutamiento con fines sexuales por grupos armados, explotación sexual en escenarios de prostitución adulta ${ }^{(1)}$. La variante callejera de la explotación sexual comercial consiste en la exposición de niños, niñas y adolescentes en la vía pública donde son abordados por adultos con fines de abusar sexualmente de ellos ${ }^{(25)}$.

En cuanto a los explotadores, se han descrito tres tipos: a) proxenetas, quienes obtienen dinero por inducir las víctimas a la explotación, actividad que realizan a través del ejercicio de la violencia o por medio de engaños, aprovechándose de la vulnerabilidad sicológica, familiar, económica o social de niños, niñas y adolescentes; b) abusadores o "prostituyentes" (mal Ilamados "clientes"); c) intermediarios, que se involucran indirectamente al prestar transporte, alojamiento u otro tipo de servicios. Aunque estos últimos actores no participan directamente en la explotación, la cohonestan, por lo que se consideran cómplices ${ }^{(26)}$.

Por el carácter clandestino de la problemática y la heterogeneidad de los actores, lugares y formas en que se desarrolla, no es posible obtener estadísticas precisas acerca de su magnitud. Sin embargo, estimaciones globales mencionan cerca de 2 millones de víctimas en el mundo ${ }^{(27)}$. A nivel regional, América Latina y el Caribe presentan la mayor prevalencia del fenómeno ${ }^{(28,29)}$. Las estimaciones para Colombia señalan de $35 \mathrm{mil}$ a 55 mil víctimas en la última década ${ }^{(30,31)}$.

Respecto a las explicaciones de la génesis de la problemática (y la permanencia de las víctimas en ella), se encuentra un buen número de modelos en la bibliografía institucional y académica ${ }^{(14,26,32,33,34,35,36)}$, los cuales, a pesar de las diferencias, coinciden en afirmar su complejidad debido a la gran cantidad de procesos y factores relacionados. Dentro de los asuntos vinculados a la explotación sexual comercial de niños, niñas y adolescentes están: alta rentabilidad de la "industria del sexo"; proliferación de actividades como el turismo y la minería sin un control adecuado; pobreza y exclusión social de familias y niños y niñas; problemáticas familiares en cuanto a estructura y funcionamiento; expulsión del sistema educativo; factores de la historia personal que generan vulnerabilidad; antecedentes en la víctima de otras problemáticas y vulneraciones de derechos; aspectos socio-culturales como el consumismo, el patriarcado y la tolerancia a la problemática ${ }^{(14,26,33,35,37,38,39,40,41)}$.

Dentro de los determinantes culturales, se destaca la imagen de la niñez entendida como inmadurez o carencia, y su subordinación a los adultos ${ }^{(42)}$. A esto se suman múltiples significados, imágenes, conceptos y representaciones sociales que tienden a normalizar o naturalizar la situación, ya sea por la tolerancia del pago por sexo como un comportamiento común y legítimo; la consideración de la explotación sexual comercial de niños, niñas y adolescentes como una actividad voluntaria de libre elección; o la aceptación de esta como una forma plausible de obtener ingresos ${ }^{(43,44,45)}$.

Ante estos aspectos culturales, la respuesta gubernamental y de la sociedad civil organizada, tanto en materia preventiva, en la atención a las víctimas o en la judicialización 
a los explotadores, se ve afectada por la manera cómo los diferentes actores significan la problemática y cómo actúan frente a víctimas y explotadores. Por ejemplo, cuando prima en actores del sector judicial una perspectiva legitimadora de la explotación sexual comercial de niños, niñas y adolescentes, se suelen subvalorar las denuncias y las pruebas judiciales para la condena a los explotadores; o también, cuando los encargados de la respuesta gubernamental conciben la explotación sexual comercial de niños, niñas y adolescentes como una forma legítima de obtener el sustento diario, es mínimo el interés para la erradicación de la problemática y se minimiza la efectiva implementación de políticas y estrategias de intervención que procuren restituir efectivamente los derechos de las víctimas.

Aunque a nivel internacional y nacional, en respuesta a esta situación, se han realizado campañas con el propósito de concientizar a funcionarios públicos y trabajadores de las organizaciones sociales acerca del carácter delictivo y lesivo de la explotación sexual para la dignidad de niños, niñas y adolescentes $^{(5,46)}$; los actores sociales y comunitarios que residen o laboran en sectores de ocurrencia de la problemática han sido poco incluidos como objetivo de estas campañas y, menos aún consultados o invitados a participar en acciones de prevención o control. Además, a nivel investigativo, poco se conoce acerca de su perspectiva frente a la situación. De esta manera, el objetivo de este artículo es discutir los principales hallazgos de un estudio cuyo objetivo fue comprender los significados y las prácticas de actores sociales que conviven con la explotación sexual comercial de niños, niñas y adolescentes en uno de los sectores de la ciudad donde la presencia de esta problemática es más evidente.

Esta investigación se anidó en un proyecto mayor titulado: "Comprensión de la explotación sexual comercial con niños, niñas y adolescentes y propuestas para su prevención y atención desde la perspectiva de este grupo de niños y niñas, Medellín, 2013-2016", el cual viene siendo ejecutado por un equipo académico de la Universidad de Antioquia (Colombia), y pretende captar la perspectiva de niños, niñas y adolescentes víctimas y otros actores relacionados, con el fin de aportar elementos y herramientas para mejorar la respuesta social y estatal con miras a la erradicación del fenómeno.

Consideramos que el abordaje de los significados posibilita entender la interacción de diferentes actores sociales y así aclarar los determinantes de las prácticas sociales, constituyendo un paso importante para la transformación de los patrones culturales y las prácticas sociales ${ }^{(47)}$, lo que se traduciría en una mayor eficacia de la respuesta ante la problemática.

\section{METODOLOGÍA}

Se realizó un estudio cualitativo basado en la propuesta de investigación social cualitativa de Galeano ${ }^{(48)}$, con el apoyo de algunas herramientas analíticas de la teoría fundamentada ${ }^{(49,50)}$. Las técnicas de recolección de información fueron la entrevista semiestructurada ${ }^{(51)}$ y la observación participante ${ }^{(52,53)}$.

Para la elección del sector de estudio, durante los meses de noviembre y diciembre de 2014 se realizaron tres salidas de exploración a seis zonas diferentes de la ciudad de Medellín, las que en un trabajo previo habían sido reportadas como escenarios de alta presencia de la problemática $^{(25)}$. La zona seleccionada se escogió por la alta visibilidad de casos de niños, niñas y adolescentes víctimas de explotación sexual, la confluencia de múltiples situaciones económicas y sociales que permitieron una visión amplia del tema de estudio y, además, las posibilidades de acceso a informantes. Así, el sector de estudio constituyó un tramo de vía pública bajo una sección elevada del metro municipal, entre dos estaciones céntricas Ilamadas "Prado" y "Parque Berrío".

En los meses de enero y febrero de 2015 se realizaron cinco recorridos en el sector de estudio para identificar algunas características sociales, las prácticas de las personas que trabajaban o residían allí, las interacciones 
de los niños y las niñas con las personas del sector, los comportamientos de transeúntes y actores estatales, entre otros. Dentro de los asuntos identificados en el sector están: múltiples locales comerciales y puestos informales de mercado, paraderos de rutas de autobuses, expendios de licor, alto número de transeúntes y la existencia de dinámicas delictivas como hurto y microtráfico de drogas.

En estos recorridos también se estableció contacto con posibles informantes y se concertó la fecha, hora y lugar de la entrevista con quienes accedieron a realizarla. La selección de los participantes estuvo guiada por criterios de conveniencia, oportunidad y disponibilidad ${ }^{(54)}$. Las entrevistas se realizaron a personas que habían permanecido en el sector de estudio, ya sea por razones de trabajo o de residencia, más de cuatro horas al día en el último año y que, adicionalmente, cumplieran los siguientes criterios de inclusión: a) referir que conocían sobre la problemática de explotación sexual en el sector; b) ser mayores de edad; c) que accedieran libremente a participar en la investigación. Se excluyeron participantes que pertenecieran a entidades del Estado como policías, empleados de programas y proyectos gubernamentales, fundaciones u organizaciones no gubernamentales. Tampoco se incluyeron transeúntes, niños o niñas, o personas bajo efectos aparentes de las drogas. Para el muestreo se utilizó la técnica de "bola de nieve" ${ }^{\prime(53)}$, para lo que se solicitó a los primeros entrevistados la referencia de otras personas del sector que pudieran participar.

Se realizó un total de ocho entrevistas, cinco a mujeres y tres a hombres. Los entrevistados se desempeñaban en el sector como vendedores informales, administradores de hoteles y de bodegas de reciclaje y trabajadores de locales comerciales.

Las entrevistas fueron de tipo semiestructuradas. Aunque se contó con un guión de preguntas, la formulación varió en función del desarrollo de cada entrevista. Además, en algunas ocasiones, se realizaron preguntas complementarias para profundizar en cuestiones relevantes que emergían en la conversación.
El guión de la entrevista se diseñó como un listado de preguntas abiertas que permitiera orientar el diálogo hacia la identificación de los significados y prácticas frente a la problemática que tenían los participantes. Las preguntas se construyeron a partir de los siguientes temas: a) experiencia de las personas en el lugar; b) identificación de problemáticas que afectaban a los niños y niñas; c) descripción de situaciones de explotación sexual y reacción propia y de otros actores frente a estas; d) características de los niños, niñas y adolescentes víctimas (como género, edad y comportamientos); e) opinión frente a la problemática y los explotadores; f) consideraciones acerca de las causas para que en la ciudad de Medellín exista la explotación sexual comercial, y motivos por los cuales las víctimas siguen inmersas en esta; g) responsables de la erradicación de los fenómenos y posibles acciones. A estas preguntas se agregó una de cierre, que indagaba por propuestas y recomendaciones.

En el Cuadro 1 se presentan las categorías que orientaron el diseño del cuestionario de la entrevista y las categorías que emergieron luego del proceso analítico. Las entrevistas fueron grabadas previa autorización de los informantes y transcritas literalmente.

La recolección de la información se hizo en paralelo con el análisis, lo que permitió ajustar progresivamente el guión de entrevista de acuerdo al proceso investigativo. El análisis de la información siguió un proceso de familiarización con los datos, codificación abierta y luego axial según una matriz de códigos realizada con las categorías emergentes. Finalmente se construyeron diagramas para representar y organizar los hallazgos ${ }^{(49,50)}$. Durante todo el proceso investigativo se realizó una revisión documental frente al tema.

Debido a las características del problema de estudio, al tratarse de una práctica en la que las víctimas son niños y niñas, la clandestinidad e ilegalidad que acompañan la problemática y las dinámicas que se viven en el sector, se mantuvo un cuidadoso manejo ético del proceso, teniendo como ejes: la integridad, responsabilidad hacia los informantes (respeto, consentimiento informado, 
Cuadro 1. Categorías orientadoras y emergentes sobre la explotación sexual comercial de niños, niñas y adolescentes. Medellín, Colombia, 2015.

\begin{tabular}{|c|c|}
\hline Categorías orientadoras & Categorías emergentes \\
\hline $\begin{array}{l}\text { - Significados frente a la problemática } \\
\text { - Características de los niños, niñas y adolescentes } \\
\text { - Comportamientos de los niños, niñas y adolescentes } \\
\text { - Relaciones e interacciones con la problemática } \\
\text { - Prácticas de las personas con niños, niñas } \\
\text { y adolescentes y la problemática }\end{array}$ & $\begin{array}{l}\text { - Significados y valoraciones contrapuestas sobre } \\
\text { la explotación sexual comercial de niños, } \\
\text { niñas y adolescentes } \\
\text { - Prácticas y comportamientos de la comunidad } \\
\text { - Prácticas de explotación y abuso } \\
\text { - Prácticas de indiferencia e indignación sin acción } \\
\text { - Prácticas de protección } \\
\text { - Limitaciones para prácticas de protección } \\
\text { por parte de personas del sector }\end{array}$ \\
\hline
\end{tabular}

Fuente: Elaboración propia.

confidencialidad, anonimato), pertinencia de las técnicas de recolección de la información y manejo de riesgos. Para ello se elaboró y ejecutó un protocolo de seguridad para el ingreso, la permanencia y el egreso del sector; igualmente, se diseñó e implementó un protocolo de consentimiento informado verbal a los entrevistados. Por razones de seguridad, no se solicitó el consentimiento informado escrito. La realización de este proyecto contó con el aval del Comité de Ética de la Investigación de la Facultad Nacional de Salud Pública de la Universidad de Antioquia, sesión 116 del 19 de febrero de 2015.

\section{HALLAZGOS}

Según lo observado, es evidente y muy frecuente en el sector la existencia de la modalidad abierta o callejera de explotación sexual. Todos los entrevistados expresaron ser testigos de casos de explotación sexual comercial, siendo común expresiones como: "eso aquí se ve a diario" (E07 pág. 24).

En este escenario, las personas que permanecen en él han construido algunos significados y valoraciones morales sobre la explotación sexual comercial de niños, niñas y adolescentes, los cuales están relacionados con diferentes tipos de prácticas respecto a las niñas y niños víctimas.

\section{Significados y valoraciones contrapuestas sobre la explotación sexual comercial de niños, niñas y adolescentes}

El proceso investigativo permitió identificar concepciones disímiles, algunas de ellas contradictorias, respecto de las situaciones de explotación sexual comercial que viven las niñas, niños y adolescentes en el sector. Para algunos entrevistados, los niños y las niñas aceptan sin presiones, o incluso propician, la demanda de adultos por tener relaciones sexuales a cambio de dinero, como lo plantea el siguiente fragmento de entrevista:

...muchos pelados o peladas [niños, niñas o adolescentes] viven por acá no es porque las obliguen sino porque ya ellas quieren vivir, si me entiende, a ellas nadie las está obligando a que tiren vicio [consuman drogas], nadie les está obligando a que se prostituyan, sino que esa es la vida que ellos quieren. (E08 pág. 29) 
Esta idea, en general, se apoya en la noción de que la explotación sexual es un libre intercambio económico, o sea, una "forma de trabajo", que permite a los niños y niñas obtener ingresos para sufragar necesidades básicas y otros gastos, como el consumo de drogas. En contraposición con esta mirada, algunos informantes aducen que las niñas, los niños y los adolescentes no tienen garantizadas sus necesidades básicas, lo que los expone a los explotadores quienes se aprovechan de su vulnerabilidad y de su estado físico y emocional para atentar contra su integridad y derechos.

Estas valoraciones definen, por un lado, la aceptación o rechazo de las personas ante la problemática, es así que si se concibe la explotación sexual comercial de niños, niñas y adolescentes como una forma de victimización, se califica como ilícita e indigna, pero si se ve como una decisión libre, ésta es legitimada, o sea, no se ve como una actividad inadecuada e ilegal.

Por otro lado, de estas valoraciones depende la mirada sobre quiénes son los responsables de que ocurra esta problemática para que se pueda resolver. De esta manera, algunas de las personas manifestaron que la responsabilidad recae en el Estado, la familia y ellos mismos como miembros de la comunidad; mientras que se encontraron manifestaciones de participantes que se excluían de esta responsabilidad, pues era una situación que solo le competía al niño, niña o adolescente que decidió estar allí. En ocasiones se ubica al Estado como único responsable de la situación y su solución.

También se pudo observar que existe una categorización de los niños y las niñas víctimas de acuerdo al tiempo de involucramiento en la explotación y el grado de deterioro físico debido a esta. De acuerdo con esta clasificación, las personas conciben que quienes han estado poco tiempo en situación de explotación son víctimas que deben recibir protección, pero en la medida que aumenta el tiempo de exposición a las situaciones de explotación, en la perspectiva de los entrevistados "que adquieren experiencia", se los responsabiliza más de su situación, al igual que los ven con menores posibilidades de recuperación. Como se verá, las prácticas tienden a la solidaridad con quienes no tienen "experiencia"; mientras que viran hacia la indiferencia u otras formas de explotación, con quienes llevan un mayor tiempo en el sector.

Estas concepciones acerca de la explotación sexual comercial de niños, niñas y adolescentes también influyen en la manera en que las personas del sector miran a quienes utilizan a niños y niñas con fines de explotación sexual. Aunque todas las personas consideraron que no está bien que un adulto explote a un niño o una niña, ya que hay una relación desigual de poder, para algunos participantes, ellos (los adultos) les permiten a los niños y niñas "ganarse" el sustento para cubrir sus necesidades básicas. De esta manera, aunque se encontraron múltiples manifestaciones de rechazo frente a los explotadores por parte de los participantes, describiéndolos como "personas muy bajas" (E03 pág. 12), "depravados" (E03 pág. 17) y "degenerados" (E08 pág. 28), no siempre los ubican como explotadores y culpables de la situación.

\section{Prácticas y comportamientos de la comunidad}

En el discurso de los entrevistados se pudo identificar cómo las anteriores concepciones conducen a diferentes tipos de prácticas en los actores que permanecen en el sector, desde prácticas de protección e indignación, hasta de indiferencia, explotación y abuso.

\section{Prácticas de explotación y abuso}

Respecto a las prácticas relacionadas con explotación sexual, pudo apreciarse diferentes formas de contacto entre los explotadores y la niña o el niño. Algunas veces el contacto se da en la calle, principalmente, en lugares donde hay aglomeración de personas. En otros casos, éste ocurre en los locales comerciales, como cafeterías y bares o en los lugares de hospedaje de niñas y niños. 
Dentro de los tipos de personas identificadas como "prostituyentes" o "clientes", se mencionaron: a) conductores de transporte público (por ejemplo, taxistas); b) "corbatas", es decir, personas con apariencia de nivel socioeconómico alto dada su forma de vestir o el tipo de auto que conducen; c) jubilados; d) turistas de diferentes nacionalidades; e) miembros de bandas delincuenciales, Ilamados "combos"; $y$, f) miembros de la fuerza pública (policías). Luego del contacto, el abuso sexual suele ocurrir en hoteles y residencias del sector o en los vehículos de los explotadores.

Otra práctica de explotación sexual reconocida es la pornografía, que ocurre en algunas viviendas del sector. Además de quienes buscan gratificación sexual, se evidencia cómo algunas personas del sector, motivados por intereses económicos, son promotores de la explotación: "antes les ayudan a que se tiren más al abismo" (E08 pág. 31). Se señala que los pares, amigos y miembros de grupos armados coaccionan a niños y niñas hacia la explotación sexual y les impiden salir de esta.

También se reportan otras formas de violencia y explotación sobre niñas y niños ejercidas por las personas del sector, tales como: a) maltratos físicos y verbales o amenazas para inducirles a la explotación; b) violencia física, verbal y sexual aun sin el interés de inducirles a la explotación; c) los inducen al consumo de drogas con el fin de utilizarlos sexualmente y como consumidores y "agentes" del mercado de drogas, ya que son principalmente ellos quienes las distribuyen y venden: "las tiran a la drogadicción para poderlas explotar..." (E3 pág. 13)

\section{Prácticas de indiferencia e indignación sin acción}

Sentir tristeza, "me da pesar" (E02 pág. 9), fue quizás una de las palabras más frecuentes en el discurso de los entrevistados, especialmente las mujeres, al referirse a la situación de niños y niñas en explotación sexual comercial. Sin embargo, este sentimiento pocas veces se traducía en acciones, debido a varias razones: a) temor; b) sensación de impotencia ante la complejidad de la problemática; c) considerar que esta situación no tiene ninguna forma de solución: "todo es un círculo que nunca se va a acabar" (E08 pág. 31).

Los sentimientos de impotencia y frustración ante la problemática, los patrones culturales que la legitiman, así como su evidencia constante en el lugar, han conllevado a que muchas personas del sector sean indiferentes y tolerantes, desviando la atención de la situación para concentrarse exclusivamente en las ocupaciones propias.

Así es como el comportamiento frente a la explotación sexual, suele ser diferente al que expresan tener frente a otras situaciones y problemáticas con las que de una u otra manera se relacionan en sus ambientes, como lo es el hurto. Este relato ilustra claramente esta situación:

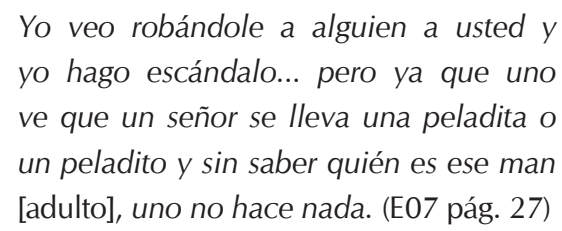

\section{Prácticas de protección}

No todas las prácticas de las personas del sector son negativas. Se evidencian algunas, aunque escasas, prácticas de protección, como: a) ayudar a niños y niñas a suplir sus necesidades básicas, "yo les doy comida... yo les presto mi casa para que se bañen... les doy ropita" (E06 pág. 22); b) intervenir frente a maltratos físicos en las calles; c) estar vigilantes a situaciones que viven ellos y ellas para buscar ayuda ante las autoridades, en especial ante situaciones críticas de salud. Estas prácticas fueron mencionadas en mayor medida por las mujeres entrevistadas.

\section{Limitaciones para el ejercicio de prácticas de protección por parte de personas del sector}

Las manifestaciones de rechazo ante la explotación sexual comercial de niños, niñas 
y adolescentes y las prácticas de protección a las víctimas por parte de las personas del sector se ven limitadas por el temor, producto de las realidades que afectan al sector, dada la "protección" que se le da a este tipo de prácticas de abuso por los pares, bandas criminales y personas que se benefician de la explotación sexual comercial de niños, niñas y adolescentes y del tráfico de drogas. Igualmente temen una posible reacción violenta de proxenetas, intermediarios o "prostituyentes".

Por otro lado, la acción de denunciar ante la policía u otros entes judiciales no es visto como una buena alternativa, debido a que la mayoría de las personas entrevistadas han perdido la credibilidad en las instituciones y en el papel que desempeñan, ya que dicen que las autoridades son conocedoras y testigos de las situaciones de abuso que viven los niños y las niñas pero no hacen ninguna acción o, paradójicamente, son ellos quienes los maltratan físicamente o verbalmente en algunas ocasiones. Es así, entonces, que la denuncia de los casos de explotación sexual es una acción que las personas participantes de la investigación han preferido obviar, siendo el silencio la principal práctica frente a la problemática.

Otro asunto que desincentiva prácticas de protección es, en palabras de un participante, considerar que "No existe ninguna forma de mejorar la situación o de ayudarles a los niños" (E08 pág. 32). Sin embargo, algunas personas también describen que han dejado de ayudar a niños y niñas, pues pese a las manifestaciones de ayuda realizadas por parte de la comunidad o del Estado continúan inmersos en situaciones como el consumo de drogas, la vida de calle, actos delictivos, entre otros. Al respecto, uno de los participantes manifestó que: "si tienen para el sacol [droga], deben de tener para comprar la comida" (E07 pág. 24). Es así como, en el concepto de los entrevistados, la respuesta de niños y niñas frente a las personas que pretenden brindarles ayuda es negativa, ya sea porque se encuentran bajo efecto de las drogas o porque mencionan que están en el sector "trabajando" y buscando dinero y que no desean protección.

\section{DISCUSIÓN}

En materia ética y jurisprudencial, la Convención Internacional sobre los Derechos de los Niños de 1989, introduce un cambio en la concepción social de la infancia: los niños deben ser reconocidos como sujetos sociales y como ciudadanos titulares de derechos ${ }^{(4)}$. Colombia, al suscribir la Convención sobre los Derechos del Niño, ha elevado a principio constitucional los compromisos adquiridos, es así como el artículo 44 de la Constitución Política indica que los derechos de los niños y niñas prevalecen sobre los derechos de las demás personas ${ }^{(55)}$. Esta norma superior, al reconocer los derechos fundamentales de los niños y las niñas, establece la obligatoriedad para la familia, la sociedad y el Estado de protegerlos contra toda forma de abandono, violencia física o moral, secuestro, venta, abuso sexual, explotación laboral o económica y trabajos riesgosos ${ }^{(56)}$. Por tanto, el cumplimiento y ejercicio de los derechos de niños, niñas y adolescentes es una responsabilidad que atañe al Estado, pero también exige de la familia y la sociedad actuar de manera activa, participativa, solidaria y concertada con el Estado, para lograr la vigencia plena de los derechos $^{(26)}$. Así, la identificación de situaciones de riesgo, su denuncia y la atención de casos de explotación sexual comercial de niños, niñas y adolescentes es una labor que debe convocar a todos los estamentos de la sociedad $^{(57)}$.

Retomando esto, entendemos que la comunidad, en especial aquella que se desenvuelve en sectores de ocurrencia de la modalidad abierta de la explotación sexual comercial de niños, niñas y adolescentes, resulta un actor fundamental para la compresión de la problemática y, sobre todo, para la intervención en pos de su erradicación. Sin embargo, hemos podido evidenciar que en el sector de estudio confluyen elementos como la cultura, las relaciones sociales, las actividades económicas legales (entre ellas, el turismo) e ilegales (como el microtráfico de drogas), además de diferentes manifestaciones de violencia y acción de bandas 
delincuenciales que enmarcan la emergencia de significados y prácticas de las personas que allí se encuentran, y que promueven condiciones para la ocurrencia de la explotación sexual comercial de niños, niñas y adolescentes.

El desarrollo de esta investigación permitió identificar concepciones contradictorias respecto a la explotación sexual y de niños, niñas y adolescentes víctimas. Aunque la mayoría de estos significados tienden a legitimar la explotación sexual comercial de niños, niñas y adolescentes, lo cual coincide con lo que la bibliografía académica e institucional ha informado acerca de la naturalización de la problemática en los escenarios donde se presenta $26,28,34,37,58,59,60,61,6$ 2,63), también se aprecian otras posturas que ponen en tensión tal naturalización como un fenómeno absoluto y evidencian algunos visos de intolerancia frente a la problemática, los cuales deben ser explorados y tenidos en cuenta por la respuesta social y estatal para la erradicación de la problemática.

Hemos observado cómo la legitimación social de la problemática conduce a considerarla como una forma válida para obtener ingresos, o sea un trabajo; a la par que no se reconoce su carácter ilegal o delictivo. Esto último, a pesar de un amplio marco normativo internacional que condena la explotación sexual comercial de niños, niñas y adolescentes e insta a los Estados a la persecución de los diferentes tipos de explotadores ${ }^{(6)}$. Esto se evidencia por las pocas denuncias que se hacen de los proxenetas, "prostituyentes" y personas involucradas en la explotación sexual comercial, así como en la poca efectividad para su judicialización ${ }^{(26)}$. Otro efecto de la legitimación social de la problemática es el poco sentido de corresponsabilidad de los actores sociales y comunitarios participantes respecto a la problemática, quienes refieren, por lo general, que quien debe actuar ante la problemática son las propias víctimas o, en menor medida, el Estado. Un ejemplo claro de ello, que reportamos en este trabajo, es la reacción diferencial frente a ser testigos de un hurto o de una situación de explotación sexual comercial de niños, niñas y adolescentes.
También, algunos autores han planteado que en el imaginario cultural hay procesos de naturalización e incluso de legitimación que afectan el interés por afrontar este delito, tal es el caso de las concepciones de habitantes del sector que refieren que la explotación nunca será erradicada pues siempre ha existido y seguirá existiendo. De acuerdo con la Organización Internacional de Trabajo (OIT), la ineficacia de las intervenciones de control contribuyen en gran medida a esta naturalización ${ }^{(59)}$, por lo tanto, es necesario insistir en la coherencia que debe tener el Estado para el cumplimiento de los mandatos normativos.

Dentro de los aspectos que el presente estudio permite relacionar con la naturalización o legitimación social de la problemática se destaca el rol que se le da a la decisión del niño, de la niña o del adolescente. De este modo, la explotación sexual suele ser aceptada cuando se considera que ingresan libre y voluntariamente de la explotación sexual comercial. Según organizaciones sociales que trabajan en el tema en Colombia, como la Fundación Renacer, este tipo de mitos se basa en una forma de validación consensual, sin crítica, que impide o retarda la toma de conciencia y la acción sobre este fenómeno, atribuyendo la responsabilidad del fenómeno a los niños y las niñas, ocultando a otros actores y factores que intervienen en el problema ${ }^{(58)}$. Sumado a esto, para Runge et al. ${ }^{(64)}$, esta idea se constituye en una forma análoga de explotación, ya que culpabiliza al niño o a la niña de la situación que vive, dejando a un lado la responsabilidad de los otros actores vinculados con esta práctica, además que se ocultan los determinantes sociales, políticos, culturales y económicos de la situación. Desde nuestro punto de vista, esta noción es una forma de ocultamiento de muchos actores que se benefician tanto económica como sexualmente de la problemática, lo que dificulta la respuesta social y estatal.

Un asunto que ha despertado nuestro interés para comprender la legitimación social de la explotación sexual comercial de niños, niñas y adolescentes es la importancia dada 
al tiempo de involucramiento en la problemática, o su "experiencia". Aunque es factible que el deterioro físico, una mayor dependencia a las drogas, el distanciamiento mayor de la familia y el reforzamiento de conductas relacionadas con la vida en calle se convierten en retos para el restablecimiento de su derechos y para los programas de re-significación de su historia de vida, esta concepción puede enmascarar complejos procesos de estigmatización y exclusión hacia grupos humanos, en este caso de niños y niñas, que por la situación en que se encuentran son socialmente "desahuciados". Al respecto, Bustelo, retomando la noción biopolítica de "homo sacer" discutida por Agamben, entendida como el ser humano que puede ser asesinado por cualquiera sin que esto sea considerado un homicidio ${ }^{(65)}$, afirma que frente a ciertos grupos de niños, que socialmente han sido catalogados como "desechables", las acciones para la preservación de sus vidas son más bien inacciones, ya que "su muerte no tiene casi ninguna consecuencia jurídica"(66). Profundizar el análisis de la explotación sexual comercial de niños, niñas y adolescentes desde la biopolítica podría ser una ruta, en futuras exploraciones, para comprender mejor los complejos procesos de naturalización que sobre esta vulneración de derechos se ciernen.

Otro asunto que tiene que ver con tal legitimación social consiste en el lugar que socialmente se le da a la dignidad humana, los derechos humanos y la legalidad, respecto al lucro. Así, cuando estos principios están en un segundo plano y prima el interés por el dinero, se tiende a tolerar cualquier actividad económica mientras sea rentable, incluso la explotación sexual comercial de niños, niñas y adolescentes. Aunque en muchos casos la necesidad de subsistencia personal y familiar se ha relacionado con la vulnerabilidad de las víctimas para caer en la explotación sexual y permanecer en ella, con lo que socialmente se ha justificado la problemática como una forma de sobrevivencia ${ }^{(35,37)}$, autores y reportes oficiales también han expuesto la ocurrencia de la problemática en niveles socioeconómicos medios y altos, con lo cual el argumento de la prevalencia del valor del dinero sobre la dignidad humana cobra importancia. Desde nuestra perspectiva, este asunto es clave en la lucha frente a la problemática y debe motivar procesos sociales y culturales amplios e intersectoriales en pos de resignificar los principios morales que rigen nuestras sociedades.

La investigación permitió observar que existe en los entrevistados una concepción de los "prostituyentes" como personas enfermas que poseen trastornos de tipo sexual y mental. Además de manera general hubo manifestaciones de rechazo frente a los explotadores por parte de los participantes. En contraste con estos hallazgos, la escasa bibliografía que trata el tema de los explotadores, hace hincapié en el hecho que no son "enfermos sexuales", sino que, en cambio, son personas adultas, con frecuencia jóvenes, que explotan sexualmente a los niños de muchas maneras distintas, por muchas razones diferentes y en diversos contextos sociales ${ }^{(58,67)}$. Según la Cátedra en Derechos Humanos de UNESCO, no se debe generalizar a los "explotadores" como pedófilos, pues quienes buscan abusar sexualmente de niños lo hacen por una amplia gama de razones y no se los puede distinguir solo por alguna característica, rasgo de personalidad, contexto social o trastorno sexual específico. En general, las únicas características en común es que todos cometen acciones que constituyen explotación sexual de los niños ${ }^{(67)}$.

Siguiendo la Cátedra, es necesario señalar que quienes demandan por explotación sexual, no lo hacen solo porque sus acciones provean alivio psíquico o gratificación sexual, sino por la permisividad de los escenarios donde se presenta la problemática ${ }^{(67)}$. De igual forma, la OIT menciona que culturalmente perviven imaginarios sociales acerca de la salud sexual de los niños y las niñas, que tienen efecto en la demanda por parte de los "prostituyentes" como, por ejemplo, que los menores de edad no contagian enfermedades de transmisión sexual o mejoran la potencia sexual, entre otros ${ }^{(61)}$. Por lo tanto, es necesario considerar que existe un vínculo estrecho entre las actitudes, significados y 
prácticas socialmente establecidos o tolerados sobre la sexualidad, el género, la raza, las clases, la niñez, la vida económica, entre otros, y la ocurrencia de la explotación sexual comercial, en las que se basan los explotadores de todo tipo para justificar y defender sus actos.

Además de los significados que hemos discutido, el desarrollo de esta investigación ha permitido apreciar que estas concepciones se vinculan a algunos tipos de prácticas de las personas del sector, ya sean de explotación, de indiferencia, de indignación sin acción y de protección. Blumer ${ }^{(68)}$, uno de los principales exponentes del interaccionismo simbólico, expone que el ser humano orienta sus actos hacia las cosas en función de lo que éstas significan para él, entendiendo la palabra "cosas" como todo aquello que una persona puede percibir en su mundo. Este planteamiento apoya lo hallado en cuanto a la influencia de las concepciones que las personas del sector tienen frente a la explotación sexual comercial de niños, niñas y adolescentes sobre las prácticas que llevan a cabo frente a la problemática y sus víctimas.

Respecto a las prácticas descritas, consideramos preciso discutir algunos asuntos. En primer lugar, los hallazgos cuestionan un poco la idea predominante en los textos académicos e institucionales de que la única repuesta por parte de la comunidad es la indiferencia, ya que se observa un panorama más amplio de reacciones que van desde la protección hasta infligir más daño.

En segundo lugar, aunque se describieron prácticas de cuidado y protección hacia niños y niñas, es claro que predomina la inacción (aunque haya indignación), otras formas de explotación y la indiferencia. En materia de intervención, resultan muy relevantes los esfuerzos por potenciar las prácticas comunitarias de protección, a la par que se intentan mitigar las limitaciones para que estas puedan ser realizadas por la comunidad, así como atenuar las prácticas lesivas y explotadoras.

En este orden de ideas, un tercer asunto, bastante preocupante, es que además de las afectaciones a nivel sexual, los niños, las niñas y los adolescentes víctimas de explotación sexual afrontan formas superpuestas de explotación económica y diversas modalidades de violencia física, mental y exclusión social. Estas situaciones evidenciadas configuran un panorama bastante adverso para los niños y las niñas víctimas, y permite argumentar que la modalidad de explotación sexual comercial callejera o abierta, en la ciudad de Medellín, dista de tratarse de una forma de transacción económica en la que hay beneficio para las dos partes, siendo más bien una clara situación de afectación de la dignidad y los derechos de los niños y las niñas, que amerita una respuesta social y estatal contundente y sostenida en el tiempo.

Por otro lado, los sentimientos de indignación descritos, nos llevan a comprender que existan casos de insatisfacción e impresión afectiva negativa sobre la problemática por parte de algunos actores del sector, pero la práctica y reacción frente a esta está condicionada y desestimulada por algunos aspectos como el temor a la acción de grupos armados, la violencia, las drogas, el dinero, o que la situación es tan grave que no va a cambiar.

En este sentido, la incertidumbre frente a las consecuencias que una acción en defensa de los niños y las niñas pueda ocasionar, sumado a la percepción de riesgo por las dinámicas de violencia y ejercicio de control por parte de bandas delincuenciales en el sector fue una constante en el discurso de los participantes. Algunos de estos condicionamientos han sido identificados en otros estudios, como el realizado por Dilacio ${ }^{(43)}$, en el que plantea que las actitudes de no involucramiento con las situaciones de explotación se construyen a través del miedo, la impotencia y una cierta indefinición en relación con las responsabilidades que las personas de la comunidad tienen con la prevención y erradicación de la problemática. De igual forma, reafirma que es un fenómeno silenciado por el temor que produce su esclarecimiento al encontrarse implicado el poder político y las personas de nivel económico elevado tanto a nivel nacional como provenientes del turismo extranjero; además de las pocas herramientas 
personales e institucionales para detectar y trabajar sobre las situaciones de explotación y en algunos casos por la percepción de que este tipo de problemáticas solo pueden ser atendidas por expertos.

Otro elemento que mina las prácticas de protección es la respuesta negativa que se ha recibido de niños y niñas, lo que puede explicarse por el efecto de naturalización de la problemática en las mismas víctimas, es decir, que ellos y ellas no siempre se conciben como víctimas de una práctica vulneradora de derechos. Esta situación puede obedecer a los crónicos procesos de agresiones sexuales que sufren, así como a los fuertes patrones culturales que legitiman la problemática. De esta circunstancia se desprenden dos grandes retos para la respuesta social y estatal. Es preciso, por un lado, realizar un intenso trabajo de acercamiento a las víctimas con la intención de propiciar la resignificación de su situación, a través de diversas modalidades de intervención tanto psicosocial como a través de las artes y el deporte; $y$, por otro lado, generar procesos decididos y sostenidos en el tiempo para hacer mella a los cimientos culturales que invisibilizan el carácter lesivo de la explotación.

Un tercer asunto que desestimula prácticas positivas por parte de la comunidad frente a la explotación sexual comercial de niños, niñas y adolescentes, como la denuncia o la protección de las víctimas, es la concepción de ineficacia de las intervenciones gubernamentales, así como la imagen de pasividad frente a la problemática por parte de la policía o, incluso, la testificación de acciones de violencia hacia niños, niñas y adolescentes por parte de la fuerza pública. Queremos convertir estos hallazgos en una exigencia a las autoridades locales y nacionales para fortalecer el control judicial y policial en los escenarios de explotación y promover la deconstrucción de significados y prácticas en estos actores que perpetúan las situaciones de vulneración para los niños y niñas víctimas.

Aunque el propósito del análisis no era hacer un comparativo de significados y prácticas desde el género de los entrevistados, se apreció que las mujeres participantes, en comparación con los hombres, expresaban de manera más abierta los sentimientos de tristeza que le generan ver las situaciones de explotación de niños y niñas y además manifestaron tener vínculos de amistad con las víctimas. También Ilamó la atención que al parecer tenían más conocimiento acerca de aspectos de tipo familiar y causas por las cuales niños y niñas estaban inmersos en la problemática. Como limitación de este trabajo, destacamos el análisis profundo desde una perspectiva de género, el cual podría abrir nuevas interpretaciones y aportar elementos valiosos para mejorar la gestión de la respuesta. Queda entonces como una tarea para siguientes trabajos la profundización de los roles de género, especialmente en cuanto a la respuesta comunitaria frente a la problemática.

Tras este estudio, podemos concluir que, en los actores sociales que permanecen en un escenario de ocurrencia de la modalidad abierta de explotación sexual comercial de niños, niñas y adolescentes en Medellín, predominan concepciones y valoraciones sobre esta problemática y las víctimas, que conducen a prácticas que agravan y perpetúan las vulneraciones de derechos, sobre otras que llevan a prácticas de protección o, al menos, de mitigación de la problemática, lo que contradice la voluntad de erradicar la problemática y contribuyen a su naturalización. Sin embargo, existen prácticas de protección en el sector para los niños y las niñas que son reflejo de la solidaridad y del papel social en la protección de sus derechos.

Frente a esto, los hallazgos de este estudio indican algunas pistas para mejorar la respuesta ante esta vulneración de derechos. En primer lugar, resulta evidente la necesidad de incluir representantes comunitarios en la planificación e implementación de acciones tanto en prevención, control y atención a las víctimas, con el fin de promover las prácticas positivas de protección y aminorar las barreras descritas para su despliegue. Asimismo, una segunda línea de actuación tiene que ver con la construcción de estrategias innovadoras que permitan la transformación de 
los significados y prácticas que perpetúan la problemática para generar conciencia de la corresponsabilidad, de la protección integral, la movilización social y la denuncia. Se recomienda también la construcción de actividades que permitan asumir socialmente al niño o a la niña en la condición de sujeto de derechos y no como objeto de atención, así como sujetos participantes, hacedores de su propia vida y no receptores pasivos.

Sin embargo, el trabajo con las comunidades de ninguna manera puede obviar la responsabilidad estatal de dar respuesta efectiva, especialmente, en el ámbito de control y protección a niños, niñas y adolescentes, máxime

\section{AGRADECIMIENTOS}

A quienes participaron con su valiosa perspectiva como entrevistados frente al objeto de estudio. A todo el equipo de investigación del proyecto de investigación "Comprensión de la explotación sexual comercial con niños, niñas y adolescentes y de propuestas para su prevención y atención desde la perspectiva de este grupo de niños y niñas, Medellín, 2013-2016", por el acompañamiento y aportes para el desarrollo de este trabajo. Agradecemos especialmente al Comité para el Desarrollo de la Investigación (CODI) de la Universidad de Antioquia, por el apoyo financiero para la ejecución de este estudio a través de la Convocatoria Pública Programática, Área de Ciencias Sociales, Humanas y Artes, año 2013. Agradecemos también a los evaluadores de las primeras versiones del manuscrito por sus valiosos aportes para el mejoramiento del texto.

\section{REFERENCIAS BIBLIOGRÁFICAS}

1. UNICEF, Cooperazione Italiana, Sistema de Integración Centroamericana. Conceptos básicos sobre explotación sexual comercial de niños, niñas y adolescentes [Internet]. Unicef [citado 31 jul 2014]. Disponible en: http://tinyurl.com/jhj9dkm.

2. Organización Internacional del Trabajo. Convenio sobre las peores formas de trabajo infantil, 1999 (núm 182) [Internet]. 2000 [citado 10 oct 2015]. Disponible en: http://tinyurl.com/zzxzbbb.

3. Krug EG, Dahlberg LL, Mercy JA, Zwi AB, Lozano $R$, (ed.). Informe mundual sobre la violencia y la salud [Internet]. Washington DC: WHO; 2002 [citado 1 oct 2015]. Disponible en: http://tinyurl.com/jz8fjtb. cuando evidenciamos cómo la percepción de efectividad gubernamental incide en el compromiso de los actores comunitarios.

En el ámbito académico se hace necesario investigar estrategias y experiencias exitosas en el empoderamiento de las familias, de la comunidad y de los diferentes actores, así como suscitar investigaciones que permitan comprender, desde diferentes perspectivas, los significados y las prácticas frente a la explotación sexual de los diferentes actores implicados en la problemática.

4. Naciones Unidas. Convención sobre los Derechos del Niño [Internet]. 1989 [citado 1 oct 2015]. Disponible en: http://tinyurl.com/jov9s35.

5. UNICEF, UNESCAP, ECPAT. Commercial sexual exploitation of children (CSEC) and child sexual abuse (CSA) in the Pacific: A regional report [Internet]. Suva (Fiji): UNICEF Pacific; 2006. Disponible en: http://tinyurl.com/j4cmjud.

6. Naciones Unidas. Protocolo facultativo de la Convención sobre los Derechos del Niño relativo a la venta de niños, la prostitución infantil y la utilización de niños en la pornografía [Internet]. 2002 [citado 1 oct 2015]. Disponible en: http:// tinyurl.com/hslcaup.

7. Organización Internacional del Trabajo, Programa Internacional para la Erradicación del Trabajo Infantil. Planos para construir un refugio: Lineamientos de un modelo de atención para niños, niñas y adolescentes en explotación sexual comercial [Internet]. OIT; 2007 [citado 30 jul 2015]. Disponible en: http://tinyurl.com/jawzqeo.

8. Organización Internacional del Trabajo, Programa Internacional para la Erradicación del Trabajo Infantil. Buenas prácticas y lecciones aprendidas: Tejiendo redes contra la explotación de niños, niñas y adolescentes - Chile, Colombia, Paraguay y Perú [Internet]. OIT; 2007 [citado 30 jul 2015]. Disponible en: http://tinyurl.com/zqb3meh.

9. Gutiérrez R, Vega L, Rodríguez EM. Problemas y dilemas éticos en la investigación de la explotación sexual comercial de niñas y niños. Salud Mental. 2008;31(5):403-408.

10. Vanegas JA, Camacho LB, Trujillo MA. La prevención de la explotación sexual comercial infantil: Un compromiso de todos. Entornos. 2009;22:95-103. 
11. Camacho LB, Trujillo MA. La explotación sexual comercial infantil: una ganancia subjetiva. Revista Latinoamericana de Ciencias Sociales, Niñez y Juventud. 2011;7(2):1009-2015.

12. Willis BM, Levy BS. Child prostitution: global health burden, research needs, and interventions. The Lancet. 2002;359(9315):1417-1422.

13. Pinzón-Rondón ÁM, Ross TWW, Botero JC, Baquero-Umaña MM. Prevalencia y factores asociados a enfermedades de transmisión sexual en menores explotados sexualmente en Bogotá, Colombia. Revista de Salud Pública. 2009;11(3):468-479.

14. Organización Internacional del Trabajo, Programa Internacional para la Erradicación del Trabajo Infantil. III Plan Nacional para la Erradicación del Trabajo Infantil y la Protección del Trabajo Juvenil 2003-2006 [Internet]. OIT; 2003 [citado 1 may 2015]. Disponible en: http://tinyurl.com/z3pncwu.

15. Weber AE, Craib KJ, Chan K, Martindale S, Miller ML, Schechter MT, Hogg RS. Sex trade involvement and rates of human immunodeficiency virus positivity among young gay and bisexual men. International Journal of Epidemiology. 2001;30(6):1449-1454.

16. Organización Internacional del Trabajo, Programa Internacional para la Erradicación del Trabajo Infantil. Explotación sexual comercial de niñas, niños y adolescentes en El Salvador. El Salvador: IPEC; 2002.

17. Fondo de las Naciones Unidas para la Infancia. Estado mundial de la infancia 2006: Excluidos e invisibles. New York: UNICEF; 2005.

18. Salaz J, Campos A. Explotación sexual comercial y masculinidad: Un estudio regional cualitativo con hombres de la población general. San José: OIT, IPEC; 2004.

19. Edinburgh L, Saewyc E, Thao T, Levitt C. Sexual exploitation of very young Hmong girls. Journal of Adolescent Health. 2006;39(1):111-118.

20. Grosfeld JL. The plight of children. Annals of Surgery. 2007;246(3):343-350.

21. Forbi JC, Onyemauwa N, Gyar SD, Oyeleye $\mathrm{AO}$, Entonu P, Agwale SM. High prevalence of hepatitis $\mathrm{B}$ virus among female sex workers in $\mathrm{Ni}$ geria. Revista do Instituto de Medicina Tropical de São Paulo. 2008;50(4):219-221.

22. Silverman JG, Decker MR, Gupta J, Dharmadhikari A, Seage GR, Raj A. Syphilis and hepatitis B co-infection among HIV-infected, sex-trafficked women and girls, Nepal. Emerging Infectious Diseases. 2008;14(6):932-934.

23. Wang EE, King S, Goldberg E, Bock B, Milner R, Read S. Hepatitis B and human immunodefi- ciency virus infection in street youths in Toronto, Canada. Pediatric Infectious Disease Journal. 1991;10(2):130-133.

24. Falb KL, McCauley HL, Decker MR, Sabarwal S, Gupta J, Silverman JG. Trafficking mechanisms and HIV status among sex-trafficking survivors in Calcutta, India. International Journal of Gynaecology and Obstetrics. 2011;113(1):86-87.

25. Muñoz-Echeverri IF, Noreña-Herrera C, Rodríguez-Garzón SA, Ossa-Estrada DA, BastidasLopera DL. Escenarios de explotación sexual comercial de niños, niñas y adolescentes, modalidad abierta, Medellín, 2012. Revista Facultad Nacional de Salud Pública. 2016;34(1):9-19.

26. ICBF, UNICEF, OIT, IPEC, Fundación RENACER. Plan de acción nacional para la prevención y erradicación de la explotación sexual comercial de niñas, niños y adolescentes menores de 18 años (ESCNNA) 2006-2011. Bogotá: ICBF, UNICEF, OIT, IPEC, Fundación RENACER; 2006.

27. International Programme on the Elimination of Child Labour, Statistical Information and Monitoring Programme on Child Labour. Every childs counts: New global estimates on child labour. Geneva: ILO; 2002.

28. Instituto Interamericano del Niño. La explotación sexual de niños, niñas y adolescentes en América Latina [Internet]. 2a ed. Montevideo: Instituto Interamericano del Niño; 2003 [citado 10 sep 2015]. Disponible en: http://tinyurl.com/h8q8fpc.

29. Save the Children. Abuso sexual infantil y explotación sexual comercial infantil en América Latina y El Caribe: Informe genérico situacional [Internet]. Bogotá: Save the Children; 2006 [citado 20 nov 2015]. Disponible en: http://tinyurl.com/ zvsbwcb.

30. UNICEF. La niñez colombiana en cifras. Bogotá: UNICEF Colombia; 2002.

31. Vargas R, Geldres D. Realidades de las niñas y adolescentes en explotación sexual del sector Prado Centro de la comuna 10 de Medellín [Internet]. Medellín: Secretaría de las Mujeres, Corporación Amiga Joven; 2012 [citado 30 jul 2015]. Disponible en: http://tinyurl.com/jhp8rc3.

32. Comité Interinstitucional Nacional. Estrategia nacional para prevenir y erradicar las peores formas de trabajo infantil y proteger al joven trabajador 2008-2015 [Internet]. Bogotá: Ministerio de la Protección Social, Ministerio de Educación Nacional, Departamento Nacional de Planeación, Instituto Colombiano de Bienestar Familia; 2008 [citado 10 jul 2015]. Disponible en: http://tinyurl. com/jc38la6.

33. Valencia Londoño DE, Botero Ramírez CA, Londoño Arredondo NH, Palacios Valencia Y. 
Factores causales de explotación sexual comercial en niños, niñas y adolescentes en la Costa Atlántica, municipios de Sincelejo y Barranquilla, con especial énfasis en población desplazada por la violencia. Medellín: Terre des Hommes Suisse, Universidad de San Buenaventura; 2008.

34. Bernal-Camargo DR, Varón-Mejía A, BecerraBarbosa A, Chaib-De Mares K, Seco-Martín E, Archila-Delgado L. Explotación sexual de niños, niñas y adolescentes: modelo de intervención. Revista Latinoamericana de Ciencias Sociales, Niñez y Juventud. 2013;11(2):617-632.

35. Instituto Colombiano de Bienestar Familiar, Observatorio del Bienestar de la Niñez. Análisis de la situación de explotación sexual comercial en Colombia: Una oportunidad para garantizar la protección de niños, niñas y adolescentes [Internet]. Bogotá: Instituto Colombiano de Bienestar Familiar, Organización Internacional para las Migraciones, Programa Presidencial de Derechos Humanos y Derecho Internacional Humanitario; 2014 [citado 30 de septiembre de 2014]. Disponible en: http://tinyurl.com/z6w9d6r.

36. Londoño NH, Valencia D, García M, Restrepo C. Factores causales de la explotación sexual infantil en niños, niñas y adolescentes en Colombia. El Ágora USB. 2015;15(1):241-254.

37. Organización Internacional del Trabajo, Programa Internacional para la Erradicación del Trabajo Infantil. Un estudio cualitativo sobre la demanda en la explotación sexual comercial de adolescentes: el caso de Colombia [Internet]. Bogotá: OIT, IPEC; 2007 [citado 15 mar 2015]. Disponible en: http://tinyurl.com/jusnmqr.

38. Organización Internacional del Trabajo. La explotación sexual comercial de niños y adolescentes: La respuesta de la OIT [Internet]. 2008 [citado 26 jul 2015]. Disponible en: http://tinyurl. com/h93zjb2.

39. Hermida MG. Características de la infancia que generan vulnerabilidad: Cartas de navegación para la prevención y erradicación de la explotación sexual comercial infantil ESCI. Bogotá: OIT, IPEC; 2005.

40. Valencia Mosquera M, Ospina Bozzi C. El abordaje de la problemática de explotación sexual infantil en Cartagena. PALOBRA, Palabra Que Obra. 2005;6(6):137-153.

41. Amar J, Madariaga C, De Castro A, Rodríguez $B$, Suárez RM. Explotación sexual comercial infantil en el distrito turístico y portuario de Santa Marta. Ter Psicológica. 2006;24(2):175-182.

42. Bustelo E. Notas sobre infancia y teoría: un enfoque latinoamericano. Salud Colectiva. 2012;8(3): 287-298.
43. Dilacio G, Giorgi V, Varela C. Las representaciones sociales de la ESCNNA y su relación con los paradigmas co-existentes en el campo de la niñez. Psicología, Conocimiento y Sociedad. 2012;2(1):170-200.

44. Gómez San Luis A. Entre la explotación sexual y la empatía: Dilemas que enfrentan los y las adolescentes. Rayuela: Revista Iberoamericana sobre Niñez y Juventud en Lucha por sus Derechos [Internet]. 2012 [citado 16 sep 2015]. Disponible en: http://tinyurl.com/h62ycva.

45. Purtscher L, Baramendi M, Farías C. Un secreto a voces: Percepciones sobre la explotación sexual comercial en Montevideo Oeste [Internet]. 2014 [citado 20 sep 2015]. Disponible en: http:// tinyurl.com/hso9dtn.

46. End Child Prostitution in Asian Tourism. What we do [Internet]. 2016 [citado 16 oct 2016]. Disponible en: http://tinyurl.com/ky5k3dh.

47. Araya Umaña S. Las representaciones sociales: Ejes teóricos para su discusión. San José: FLACSO; 2002.

48. Galeano ME. Diseño de proyectos en la investigación cualitativa. Medellín: Fondo Editorial Universidad EAFIT; 2004.

49. Charmaz K. "Discovering" chronic illness: using grounded theory. Social Science and Medicine. 1990;30(11):1161-1172.

50. Strauss A, Corbin J. Bases para la investigación cualitativa: Técnicas y procedimientos para desarrollar Teoría Fundamentada. Medellín: Facultad de Enfermería, Universidad de Antioquia; 2002.

51. Charmaz K. Grounded theory in the 21st century: Applications for advancing social justice studies. In: The SAGE Handbook of Qualitative Research. 3a ed. Thousand Oaks, California: Sage Publications; 2005. p. 507-535.

52. Guber R. La etnografía: método, campo y reflexividad. Buenos Aires: Editorial Norma; 2001.

53. Taylor SJ, Bogdan R. Introducción a los métodos cualitativos de investigación: la búsqueda de significados. Barcelona: Paidós; 1987.

54. Sandoval Casilimas CA. Investigación cualitativa. Bogotá: ICFES, Asociación Colombiana de Universidades e Instituciones Universitarias Privadas; 1996.

55. República de Colombia. Constitución Política de Colombia [Internet]. 1991 [citado 10 sep 2015]. Disponible en: http://tinyurl.com/zesr3c9.

56. Ministerio de la Protección Social, Ministerio Nacional de Educación, Instituto Colombiano de Bienestar Familiar. Documento CONPES social 
Política Pública Nacional de la Primera Infancia "Colombia por la primera infancia" [Internet]. 2007 [citado 10 sep 2015]. Disponible en: http:// tinyurl.com/p5rlld2.

57. Díaz Benjumea M, Blandón Loaiza LM. Representaciones sociales de explotación sexual comercial de niños, niñas y/o adolescentes-ESCNNA, en el corregimiento de la Boquilla. Medellín: Universidad de San Buenaventura; 2009.

58. Plan Internacional, Fundación Restrepo Barco, Fundación Renacer. Escenarios de la infamia: Explotación sexual comercial de niños, niñas y adolescentes. Bogotá: Plan Internacional, Fundación Antonio Restrepo Barco; 2006.

59. Joves Rueda ME. Navegar con rumbo: Cartas de Navegación para la Prevención y Erradicación de la Explotación Sexual Comercial Infantil -ESCI [Internet]. Bogotá: OIT, IPEC; 2006. Disponible en: http://tinyurl.com/n5a9frm.

60. Grajales N. Dimensiones cualitativas de la explotación sexual infantil en la localidad de Chapinero: Límites de la política pública distrital y alternativas de intervención. Papel Político Estudiantil. 2008;4(1):191-212.

61. Guaqueta Rodriguez C. ESCl: Prácticas culturales que la promueven - Cartas de navegación para la prevención y erradicación de la Explotación Sexual Comercial Infantil ESCI. Bogotá: OIT, IPEC; 2005.

62. Morales SA. Explotación sexual infantil y juvenil: una mirada a los sectores y actores en la ciudad de Medellín. En: Construcción interactiva de ambientes para el desarrollo humano: rompiendo círculos de explotación sexual infantil y juvenil "Acercamientos metodológicos y teóricos". Medellín: FUNLAM, Centro de Investigaciones; 2002. p. 78-104.

63. United Nations Office on Drugs and Crime, Alcaldía de Medellín. Estudio exploratorio descriptivo de la dinámica delictiva del tráfico de estupefacientes, la trata de personas y la explotación sexual comercial asociada a viajes y turismo en el municipio de Medellín, Colombia [Internet]. UNDOC, Alcaldía de Medellín; 2013 [citado 20 sep 2015]. Disponible en: http://tinyurl.com/ j24uu3n.

64. Runge AK, Piñeres JDP, Hincapié A. Subjetivaciones, lenguaje y parodia: reflexiones en torno a los discursos expertos sobre la Explotación Sexual Comercial de Niños, Niñas y Adolescentes (ESCNNA). Revista Latinoamericana de Ciencias Sociales, Niñez y Juventud. 2010;8(1):269-291.

65. Agamben G. Homo Sacer: El poder soberano y la nuda vida. Valencia: Pre-Textos; 1998.

66. Fundación Renacer. ESCNNA: Conceptos [Internet]. Bogotá: Fundación Renacer; 2011 [citado 12 may 2015]. Disponible en: http://tinyurl.com/ zmq8vcq

67. Cátedra UNESCO de Derechos Humanos. El explotador sexual [Internet]. Biblioteca Educación a Distancia en Derechos Humanos [citado 20 may 2015]. Disponible en: http://tinyurl.com/zy4cgyr.

68. Blumer H. El interaccionismo simbólico: perspectiva y método. Barcelona: Hora; 1981.

\section{FORMA DE CITAR}

Ossa-Estrada DA, Muñoz-Echeverri IF. Explotación sexual comercial de niños, niñas y adolescentes: significados y prácticas de trabajadores/as y residentes del centro de Medellín (Colombia), 2015. Salud Colectiva. 2017;13(1):19-34. doi: 10.18294/sc.2017.1107.

Recibido: 26 de agosto de 2016 | Versión final: 21 de octubre de 2016 | Aprobado: 1 de noviembre de 2016

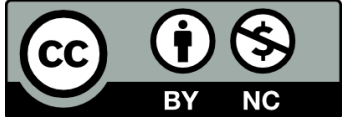

Este obra está bajo una licencia de Creative Commons Reconocimiento-NoComercial 4.0 Internacional. Reconocimiento - Permite copiar, distribuir y comunicar públicamente la obra. A cambio, se debe reconocer y citar al autor original. No Comercial - Esta obra no puede se utilizada con finalidades comerciales, a menos que se obtenga el permiso.

http://dx.doi.org/10.18294/sc.2017.1107 